\title{
Framework of models for selecting manufacturing processes and associated parameters for surface topographies
}

\author{
Benoit Rosa ${ }^{1, *}$, Maxence Bigerelle ${ }^{2}$, Antoine Brient ${ }^{1}$, and Serge Samper ${ }^{1}$ \\ ${ }^{1}$ Institut de Physique de Rennes (IPR), UMR CNRS 6251, Rennes, France \\ ${ }^{2}$ Laboratoire d'Automatique de Mécanique et d'Informatique Industrielles et Humaines (LAMIH), UMR CNRS 8201, \\ Valenciennes, France
}

Received: 7 December 2016 / Accepted: 19 February 2019

\begin{abstract}
Choosing appropriate manufacturing processes to create functional surfaces is a challenging issue for some industrials. A specific surface finish can be obtained by different manufacturing processes, each of them having a different economic impact. Currently, no tool could guarantee the surface function through the choice of a manufacturing process and its associated operating parameters. This paper aims at discussing about a framework of models for selecting conventional or innovative manufacturing processes and their associated parameters with regards to surface topographies and textures. To achieve this, a concept of decomposition of database is introduced. Manufacturing processes such as, electro discharge machining, water jet machining (used for texturing surfaces), sandblasting and laser cladding are modelled. Finally, a concept that links such a database with computer aided design (CAD) software in order to integrate surfaces functionalities and manufacturing processes directly into the design step is discussed.
\end{abstract}

Keywords: Manufacturing processes / operating parameters / modeling / topography / data selection

\section{Introduction}

Functional surfaces (i.e. surfaces that ensure a functionality) can be either natural or engineered (Fig. 1). Naturally textured surfaces can have various functionalities such as hydrophobic behaviour or biological properties. Human specifies and manufactures surfaces in order to obtain functionalities essential for specific applications (tribological, aerodynamical drag reduction, wear...). Sometimes, it is also worth creating nature-inspired surfaces through manufacturing processes. Yin et al. [1] have developed a super hydrophobic surface based on nature inspired functionalities (e.g.: water droplets rolling on a lotus leaf, self-cleaning wings of butterflies, superhydrophobic rose petals with high liquid adhesion...) using sandblasting and chemical etching processes.

This study shows the relationship between operating parameters settings and the final functionality. More precisely, the $\mathrm{NaOH}$ solution and the etching time are impact on the porosity of the surface and finally on its hydrophobic performance.

Nature inspired surfaces are based on natural model and allow the replication of a natural functionality into a

\footnotetext{
* e-mail: benoit.rosa@ec-nantes.fr
}

mechanical system (shark skin texture for hydrodynamic applications for example). Theses surfaces can often be achieved using various manufacturing processes.

Manufacturing of functional surfaces requires knowledge and skills in many different areas. Choosing a manufacturing process and the associated manufacturing parameters that lead to a specific surface finish requires to know the texturing capabilities of numerous conventional and innovative processes and the influence of their manufacturing parameters on the surface.

In addition, a perfect control of the relationship between the surface integrity, its preservation, and the workpiece functionalities is necessary. Since surface topography can be characterized at different scales, it is mandatory to know how the efficiency of a surface functionality evolves with regards to the different scales. In this context, care must be taken to assess the surface using relevant amplitude, spatial and/or texture parameters.

Building a tool that could help choosing a means to manufacture a functional surfaces is challenging since it requires to model the relations between function, surface and process in various application areas. A few studies have been focused on the correlation between surface characteristics and their function [2-4]. Other studies have investigated the influence of the process parameters on the surface finish [5-7]. 


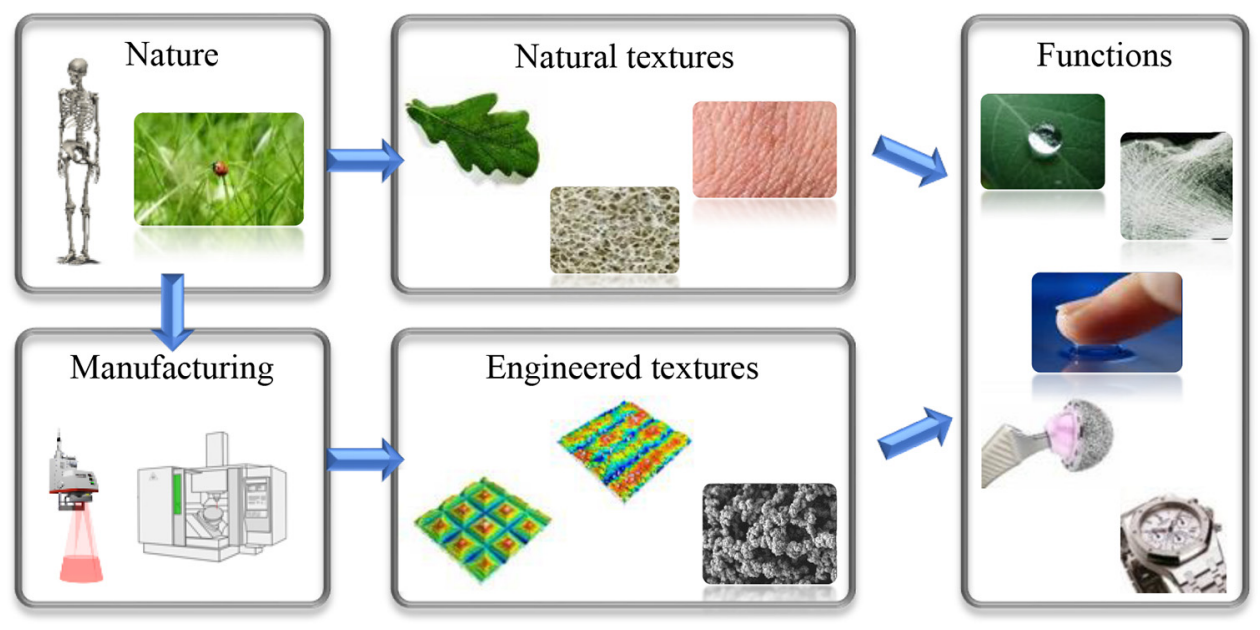

Fig. 1. Global surface context.

Some studies have also been carried out on topographies classification, but no correlations with manufacturing processes have been established yet [8]. These studies consist mainly in large database of measured surfaces with their associated manufacturing processes and parameters, established in order to help industrial companies choosing a process. No modeling of the relations between surface parameters and process parameters is actually integrated nor proposed [9].

This paper aims at proposing a framework of models for obtaining specific surface topography by selecting manufacturing processes and their associated parameters. Built up through experimentations and statistical tool, the models would establish the relationship between operating parameters and associated surfaces.

In parallel, in order to guarantee the final quality of parts through the numerical manufacturing chain a concept of a functional CAD surfaces that integrate the proposed framework database is introduced.

\section{Problem definition}

The decision-making tool has to integrate the processes surfaces - functionalities relations and as such it depends on different parameters (multiscale surface parameters and operating parameters) and involves a complex database (Fig. 2).

The relations between parameters can be summarized as an input / output system. The input is the surface function i.e. the specification of the part. The outputs are the manufacturing process and its associated parameters. It involves that the user knows the relationship between function and surface characteristics.

The database is built using both empirical and modeling approaches. Several measured samples integrated in the database would directly provide the manufacturing parameters if their surface characteristics are the expected result. To increase the spectrum of potential surface machining parameters, additional manufacturing models would be integrated.
Several mathematical methods exist to predict desired surface for operating parameters setting, such as numerical simulation [10] or statistical tools [11].

Numerical simulation is based on physical phenomenon equations. The creation of a measured database is not necessary. All predictions are based on physical phenomenon and not from boundaries measured data. However, calculation times are very important and tend to limit its use. For laser based processes, simulation of the metal flow phenomenon with regards to thermic evolution is complex, and the calculation times even for a small area make it hard to be used efficiently. For conventional processes such as milling process however, some studies propose a robust simulation of the final surface regarding operating parameters [12]. The choice of a method would depend on the physical principle of the manufacturing process.

With their shorter calculation times, the statistical methods are more appropriate in an industrial context. Artificial neural network (ANN) had a high efficiency and strength regarding modelling and predicting [13]. However, the ANN method results in a "black box" model that tends to limit the interpretation of results and the knowledge of the studying system.

In order to decrease calculation time, a polynomial regression method could also be achieved [14]. This mathematical tool enables representing visually the manufacturing processes behavior and help improving its understanding. For these reasons, the regression method has been used in this study.

\section{Methodology of investigation}

\subsection{Decomposition of database concept}

In order to find the optimal machining parameters with regards to the surface functionality, a methodology based on the database decomposition is proposed (Fig. 3). The database is divided into three parts. The first part consists in modelling the relationship between manufacturing processes and the associated surfaces through a statistical approach based on experimentations. The models enable to 


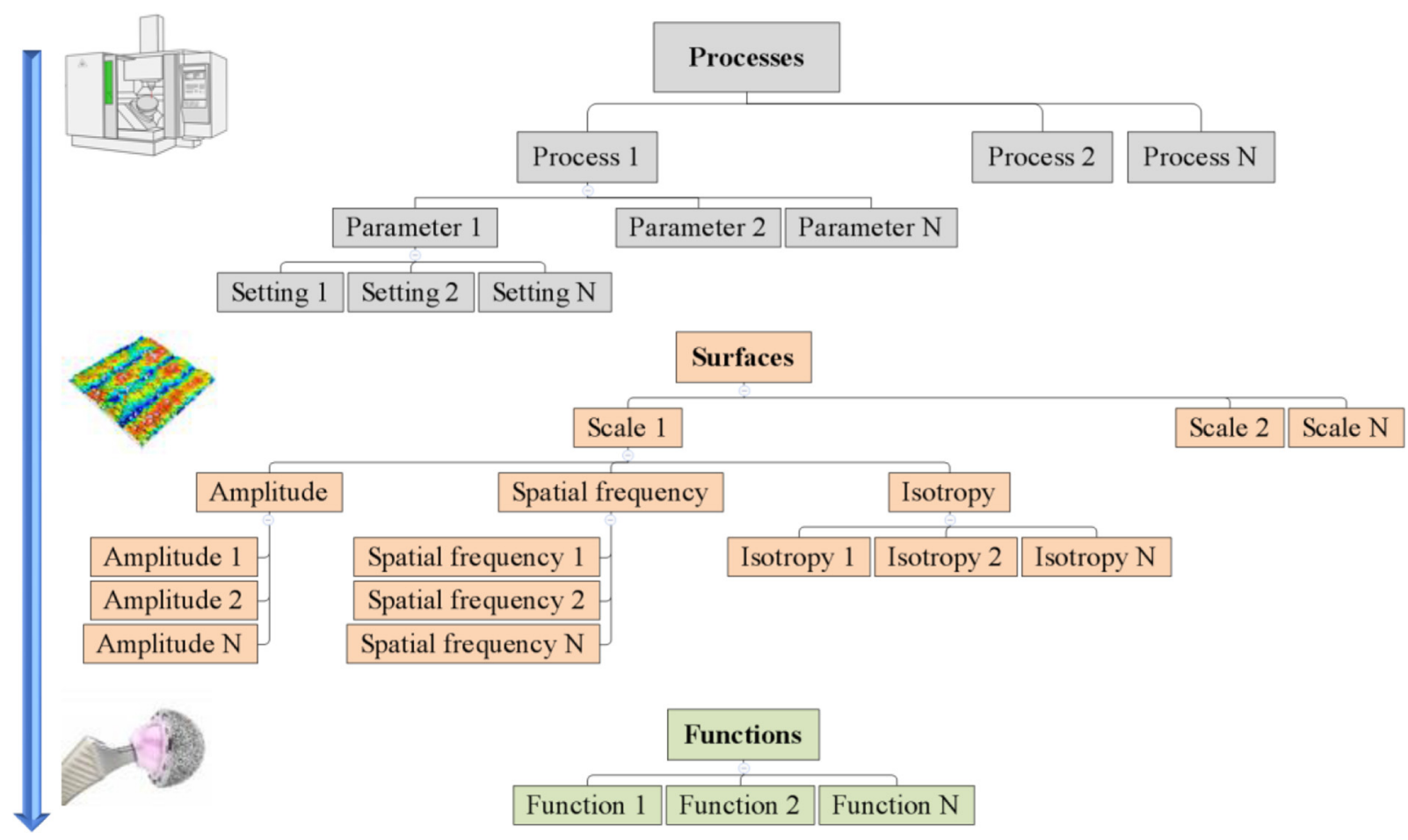

Fig. 2. Conceptual schema of database.

identify the surfaces texture with regards to operating parameters for some manufacturing processes. This modelling part leads to a first organized framework which aims at proposing manufacturing process with regards to the desired surface texture. The framework is based on the operating parameters models.

The second part consists in modelling the relationship between surfaces and functionalities and similarly leads to a second framework that links surfaces to functions.

The third part is the definition of the final framework that combined the two previous frameworks and contains the manufacturing processes and associated operating parameters - multi-scale surfaces - functionalities.

The multiscale methods allow to analyse surface measurements at every available scale, the finest scale limit is formed upstream when measuring the surface (choice of the $\mathrm{x}$-axis and $\mathrm{y}$-axis spacing). The authors have developed a method to find the best relevant scale and have tested three multiscale methods on 14 tribological processes (a normalised gaussian filtering, a discrete wavelet transform and the more recent discrete modal decomposition) [15].

The basic principle consists in carrying out $X$ multiscale decomposition methods with different $P$ passes (high pass, low pass and band pass filtering on $E$ scales) on the $I$ surfaces measured for each of the $C$ configurations of the studied process which gives a map $M(x, p, e, i, c), x \in X$, $p \in P, e \in E, i \in I, c \in C$. From each Map $M(x, p, e, i, c), Q$ roughness parameters are calculated written $R(q, x, p, e, i, c)$. Let $F(i, c)$ be one (or more) surface functionalities obtained on the $i$ th tooled surface with a configuration $c$ of the process. Let $\psi$ be a function taken from a set $\psi$ which gives a possible relation between a roughness parameter $q$ and $a$ function $F$ and let $\chi$ another function $(\chi \in \chi)$ which gives a possible relation between a roughness parameter $q$ and the $C$ configurations process (structuring). Note $\Theta$ a standardized function of relevance of the selected model. So, the search for a relevance roughness parameter is to solve the following optimization problem:

Find $\left(q_{\text {opt }}, x_{\text {opt }}, p_{\text {opt }}, e_{\text {opt }}, c_{\text {opt }}, \chi_{\text {opt }}, \psi_{\text {opt }}\right)$ such MAX $\left\{\alpha \Theta(\boldsymbol{\psi}(q, x, p, e, c, \psi))+(1-\alpha) \Theta^{\prime}(\chi(q, x, p, e, c, \chi))\right\}, \alpha \in[0,1]$.

If $\alpha=1$ then the roughness parameter (at its optimal multi-scale decomposition) characterizes the relationship between surface topography and functionality [16].

If $\alpha=0$ then the roughness parameter (at its optimal multi-scale decomposition) characterizes the relationship between the parameters of the manufacturing process and the surface topography [17].

Otherwise, $\alpha$ represents a compromise between the surface functionality ( $\alpha$ large) and the surfaces texturing ( $\alpha$ small) $[18,19]$.

The proposed study is focused on the modelling of operating parameters with regards to associated surfaces for three different manufacturing processes, and the creation of a first model based framework.

\subsection{Experiments}

The investigations are based on experiments and focuses on three manufacturing processes: electro discharge machining (EDM), water jet machining (WJM) and laser cladding (LC). Experiments take into account the operating parameter variation for each process as can be seen in Table 1. For EDM, the operating parameters are not mastered during the experiments. The interface of the EDM machine does 


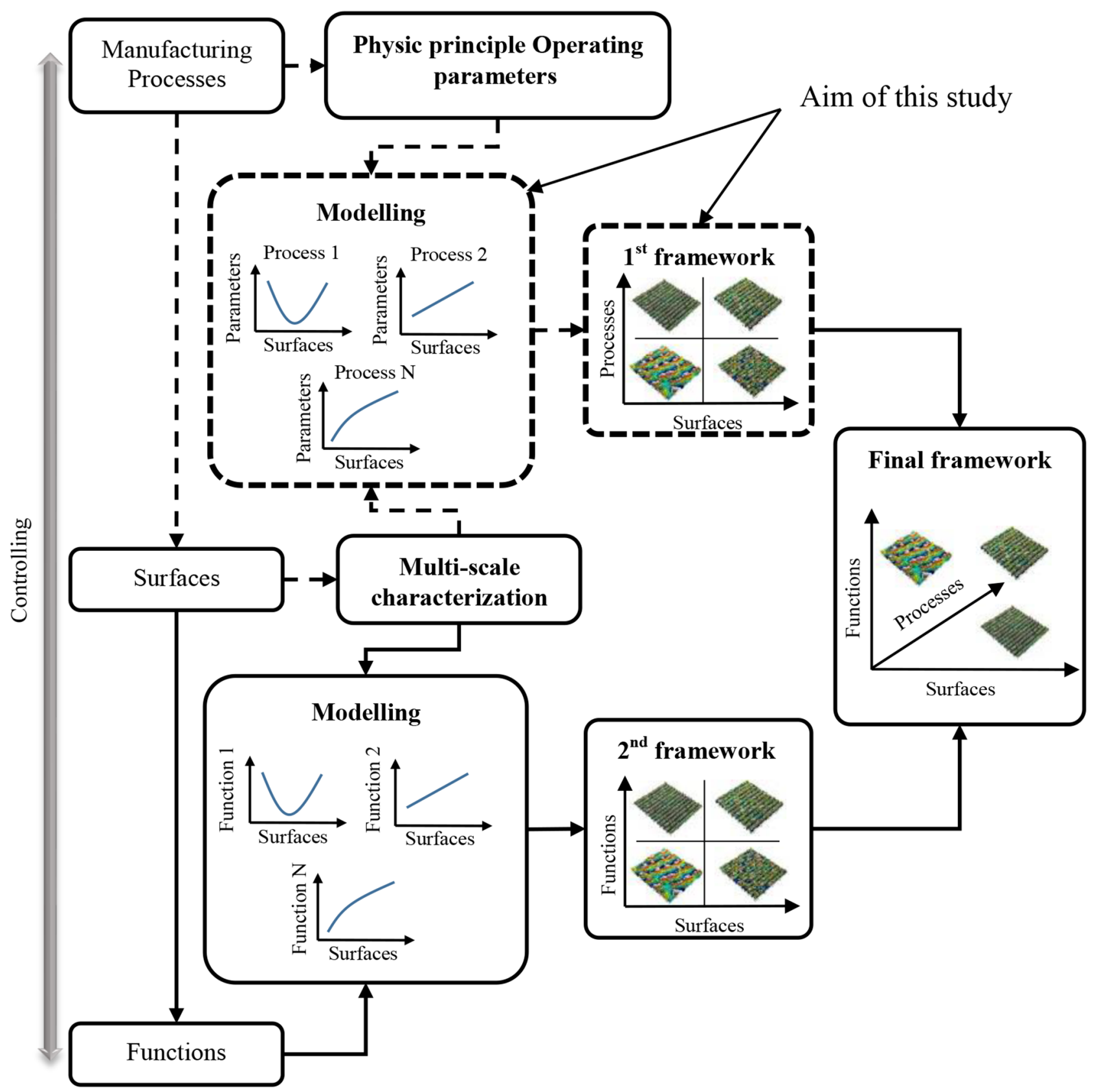

Fig. 3. Methodology for database decomposition.

not allow operating parameter variation but gives a supposedly achievable roughness average as a global parameter. For this particular case, the surface roughness is therefore the input data. The offset parameter was investigated for WJM. The offset parameter is the distance between the focal point of the water jet and the machined surface. The offset parameter influences the water jet strain and its distribution density. In the case of laser cladding, laser track step has been investigated. Laser track step $\left(L_{t s}\right)$ is the distance between two deposited material tracks. An $L_{t s}$ equal to 0 leads to building walls of material.

\subsection{Surface characterization}

The surface texture impacts the surface functions. In order to understand and control laser cladded surfaces, 25178 standard ISO [20] parameter is used in order to characterize the first level texture: Str parameters. Based on autocorrelation, Str parameter, the texture aspect ratio, is a measure of the spatial isotropy or directionality of the surface texture (Fig. 4). For a surface with a directional lay, the Str parameter tends towards 0 whereas a spatially isotropic texture result in an Str of 1 . 
Table 1. Processes and operating parameters ranges of experiments.

\begin{tabular}{lll}
\hline Processes & Operating parameters & Ranges \\
\hline EDM & Surface roughness $\left(R_{a}\right)(\mu \mathrm{m})$ & $3.2-18$ \\
WJM & Offset $\left(O_{f}\right)(\mathrm{mm})$ & $3-14$ \\
LC & Laser track step $\left(L_{t s}\right)(\mathrm{mm})$ & $0.2-0.7$ \\
\hline
\end{tabular}

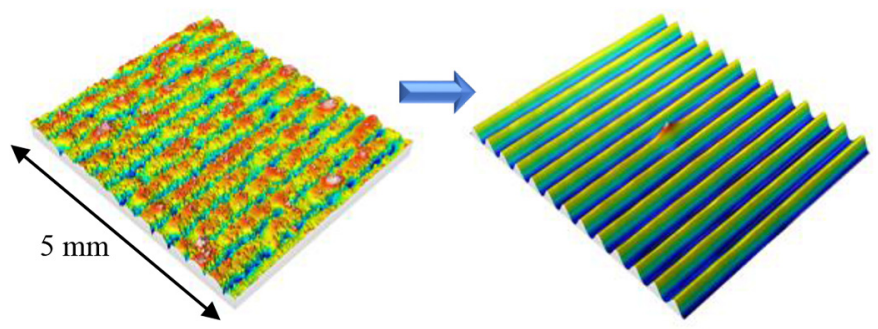

Fig. 4. Surface topography (left) and associated surface texture isotropy (right) obtain by autocorrelation.

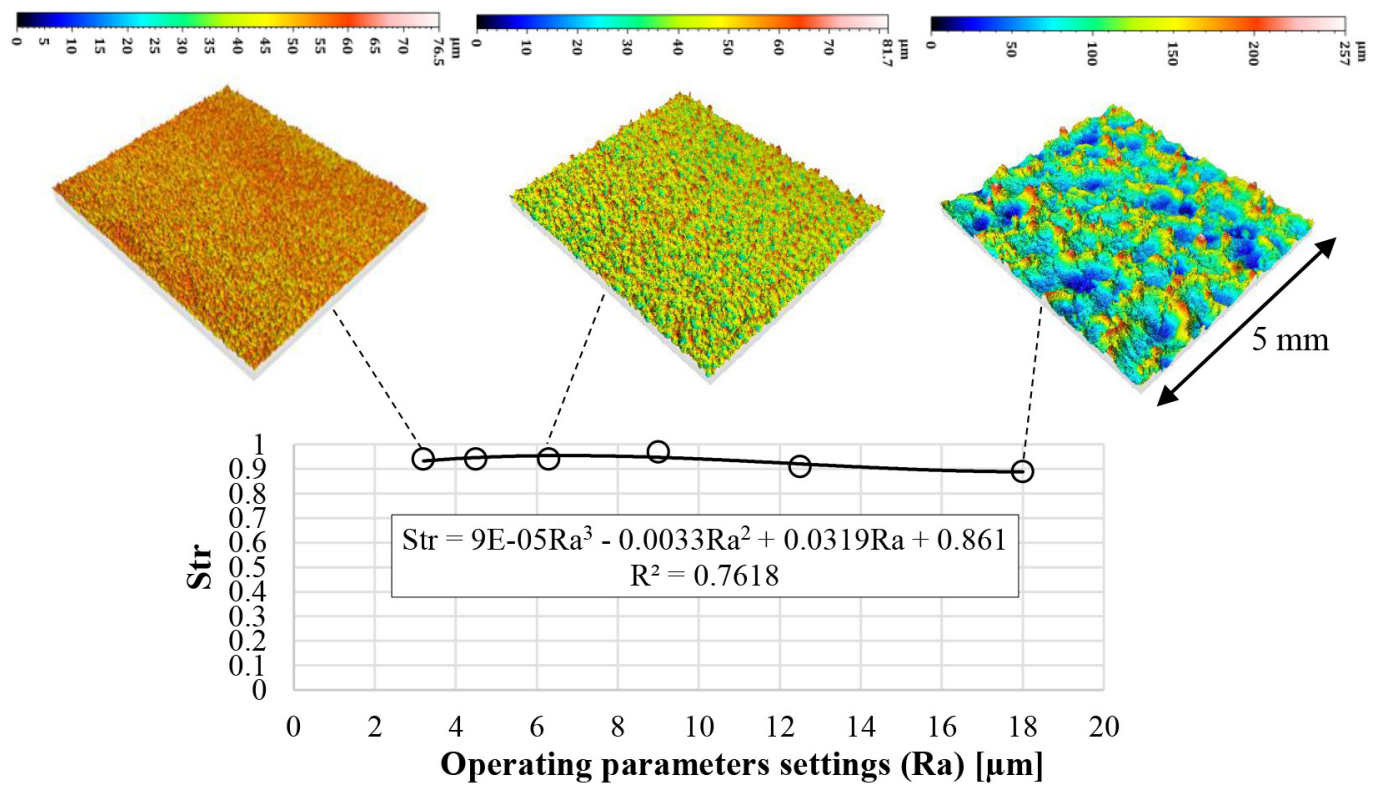

Fig. 5. EDM operating parameter behaviour regarding surface texture (Str parameter).

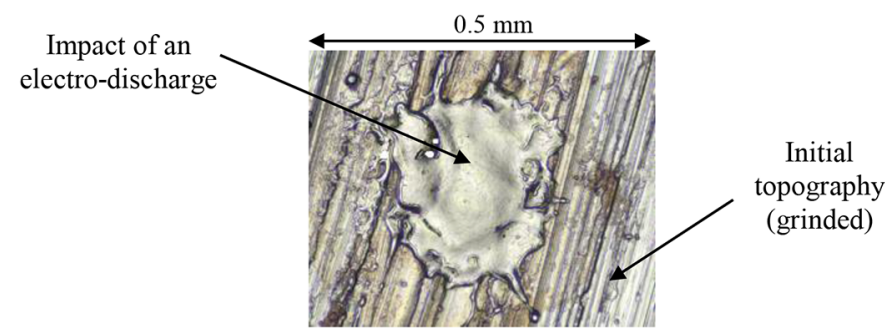

Fig. 6. Impact of one electro discharge of EDM process.

\section{Results}

\subsection{Electro-discharge process modelling}

For EDM process, the variation of operating parameters does not influence the Str parameters values. Globally, the obtained surfaces are anisotropic (Fig. 5). This phenomenon is directly caused by the physical principle of the process. During the process, electric discharges are uniformly disposed on the electrode surface. Each electro discharge creates a material sublimation and fusion (Fig. 6) that is uniformly spreads on the treated surface and finally tends to create an anisotropic texture. In these conditions, the EDM process does not allow to obtain directional surface texture.

\subsection{Water jet machining process}

For WJM process the variation of the offset parameter impacts on the surface texture isotropy (Fig. 7). The model behaviour shows that increasing the offset parameter value tends to increase Str parameter and to create an anisotropic surface texture. The proposed model is correlated at $96 \%$ with the experimental data. Globally, the water jet machining process enables to create an Str situated between 0.4 and 1. Decreasing the offset parameter value tends to increase water jet strain. In this case, some material is removed which tends to create some directional grooves over the surface with regards to the path direction. In parallel, the increase of the offset parameter value tends to decrease water jet 


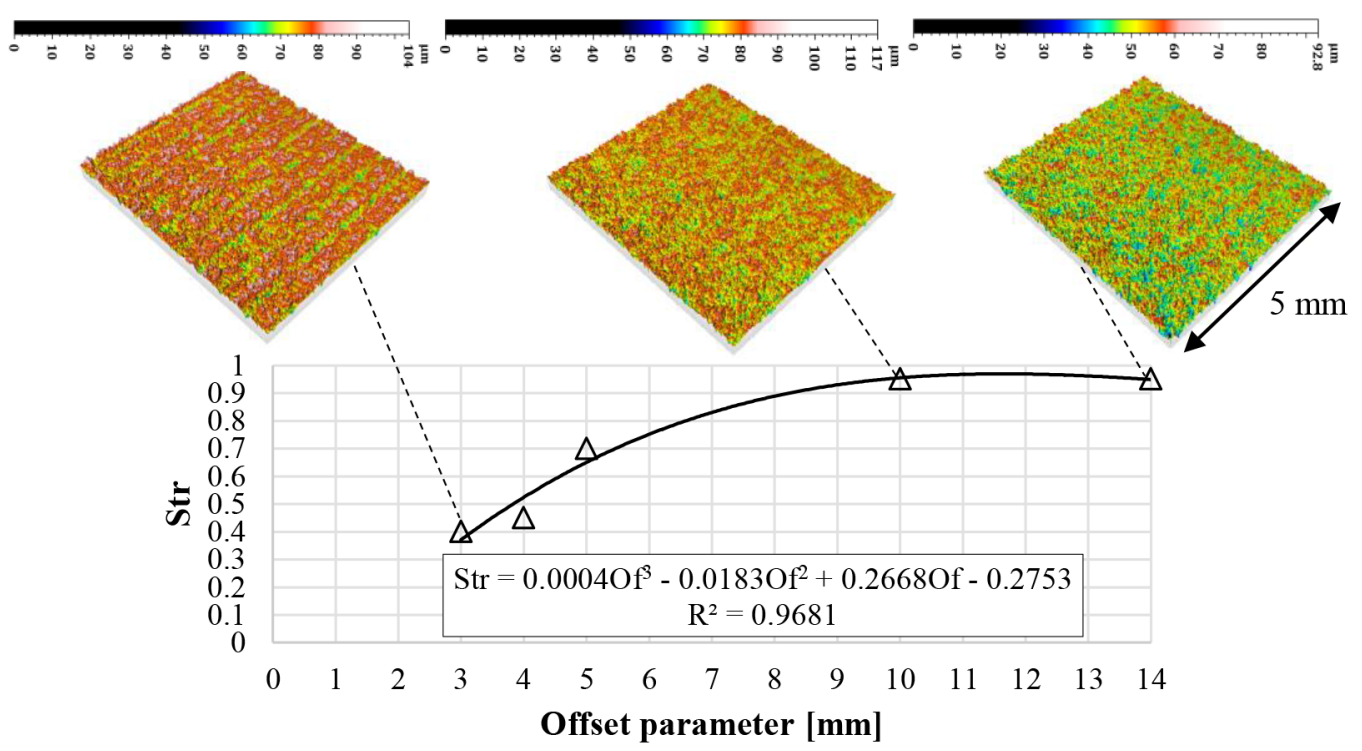

Fig. 7. Modeling of WJM operating parameter regarding surface texture (Str parameter).

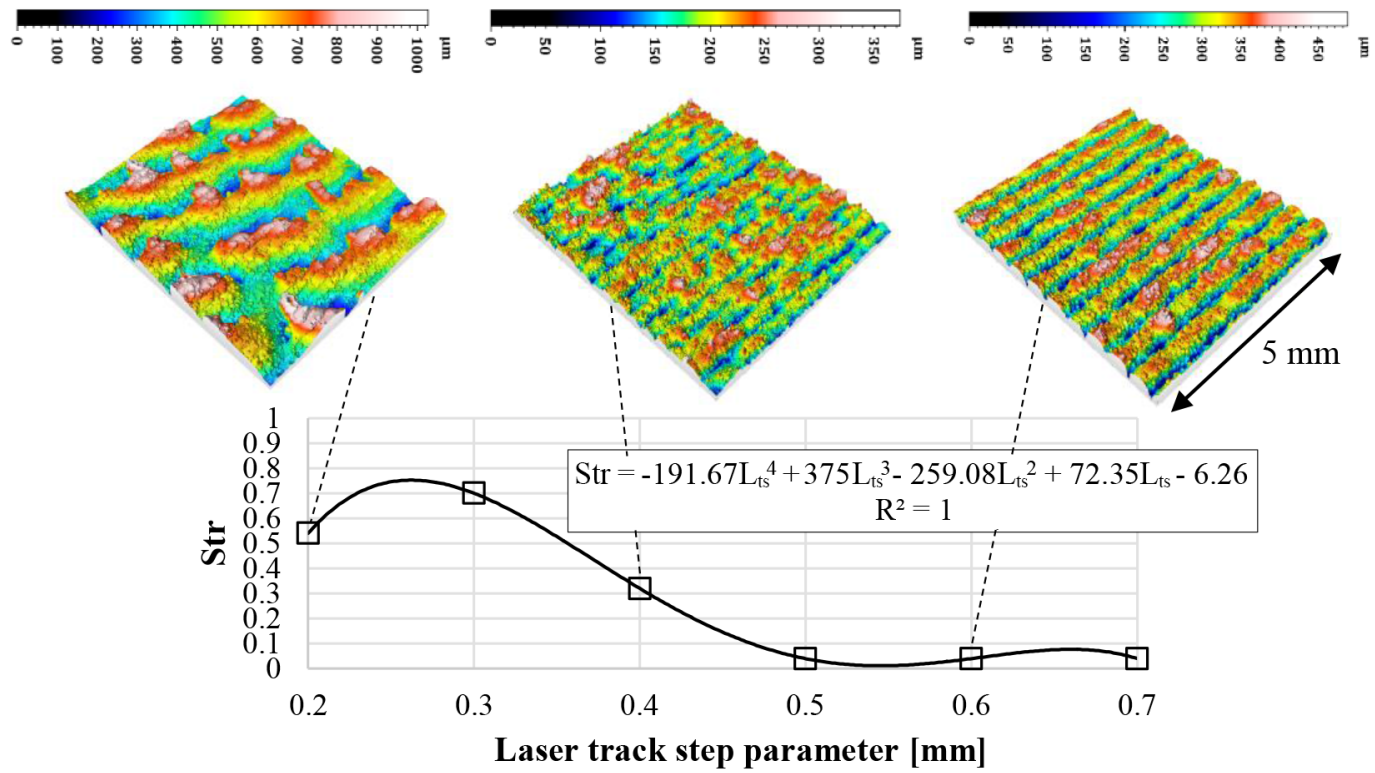

Fig. 8. Modeling of LC operating parameter regarding surface texture (Str parameter).

strain. In these conditions, the removed material is decreased and no grooves are created. The increase of offset tends to create a directional texture like a sandblasted surface.

\subsection{Laser cladding process modeling}

For laser cladding process, the laser track step enables to create a surface texture with an Str value under 0.7. The proposed model is perfectly correlated with the experimental data. Globally, the decrease of laser track step tends to increase the Str parameter. This phenomenon is caused by the superposition of deposit material that tends to influence the texture direction (Fig. 8). Under a 0.5 laser track step value, the texture is only directional. In this case, there is no overlapping of material deposit tracks, and the texture is directional with regards to the path direction.

\subsection{Framework of models for manufacturing processes selection}

Regarding operating parameters models, it is possible to define a framework of models (Fig. 9). The proposed framework allows the identification of the most relevant processes with regards to the desired surface texture. In parallel, this framework diagram enables to select the operating parameters and their settings in order to guarantee the desired surface texture isotropy. 


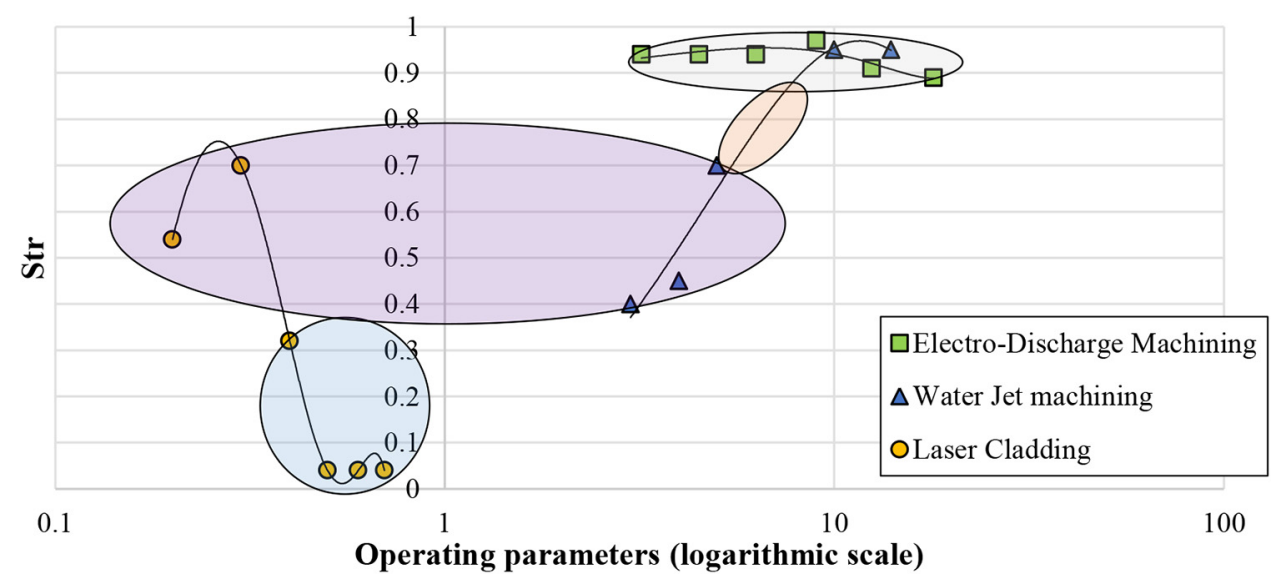

Fig. 9. Framework of models for selecting manufacturing processes and operating parameters for surface isotropy.

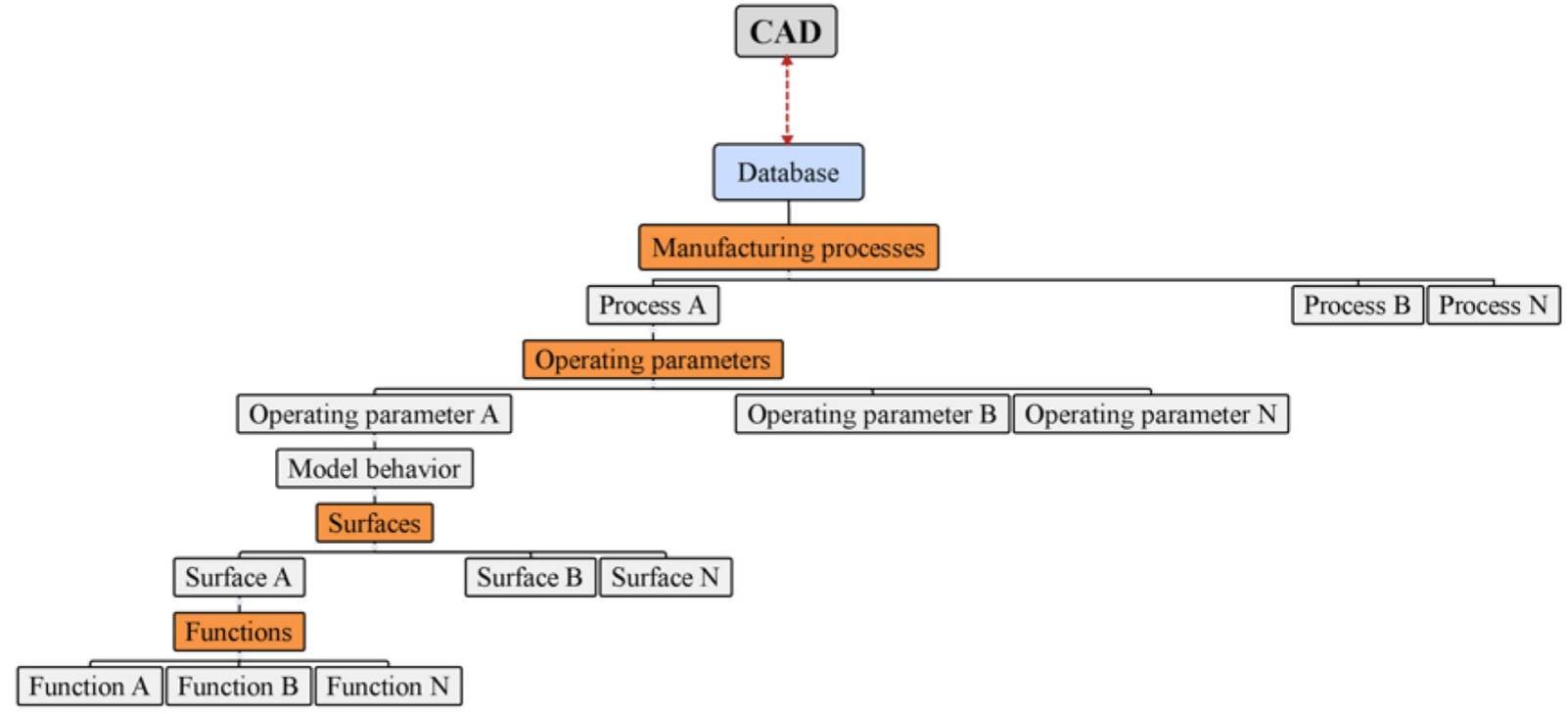

Fig. 10. Functional CAD concept based on database.

According to the diagram's framework, both electro discharge machining and water jet machining processes enable to obtain the same texture anisotropy. Laser cladding and water jet machining enable to obtain the same surface texture Str between 0.8 and 0.4. In an industrial context, it is possible to determine the least expensive process for a specific surface texture. In parallel, it is possible to improve manufacturing process planning by choosing actually available manufacturing means. This proposition of tool enables to improve the productivity and the determination of manufacturing processes according to the surface texture.

\section{Towards a surface function integrated CAD/CAM system}

The ultimate goal of this work is to create a surface function integrated CAD (Computer Aided Design)/CAM (Computer Aided Manufacturing) system that relies on an open source database of processes and functions (Fig. 10). For now, while surface parameters can be specified in parts detailed drawings they do not provide information on the expected function. Regarding the manufacturing process, defining the operating parameters values is mainly achieved experimentally and if few models are used to guaranty the surface finish, none of them take into account the final function of the surface.

The proposed concept would feature models that correlate surface finish with manufacturing operating parameters and models that correlate surface finish with functions. It would allow to define an appropriate manufacturing process and its operating parameters with regards to the expected function. Since a specific surface finish can be achieved by different manufacturing means, the surface function integrated CAD/CAM system would provide the user with a set of alternative processes and could also handle a multi-processes approach. All the manufacturing data will be integrated into the numerical chain from the CAD file to the manufacturing process. 


\section{Conclusions and further researches}

This paper focused on surface classification for manufacturing processes. Based on experimentations, some operating parameter models with regards to the texture isotropy are proposed for different manufacturing processes. The obtained models are well correlated with experimental data and improve knowledge of the operating parameters behaviour. As a result, the issued classification enables to determine a manufacturing process that allows to obtain the desired surface texture in order to guarantee the surface functionality. Within the range of this study some conclusions can be established:

- DMD process enable to obtain anisotropic texture Str situated around 1 ;

- WJM process enable to obtain Str situated between 0.4 and 1 by choosing an appropriate value of the offset operating parameter;

- LC process enable to obtain Str situated between 0 and 0.7 by choosing an appropriate value of the laser track step operating parameter;

- some manufacturing processes enable to obtain the same texture isotropy;

- the proposed framework allows to determine the most appropriated process regarding surface texture, cost and availability.

Further researches will focus on the integration of other manufacturing processes, other operating parameters relevant of the studied processes and their interactions. Indeed, the integrity, others scales of textured surfaces and functionalities must be taken into consideration.

Acknowledgments. The authors would like to thanks Pr. JeanYves Hascoët of the École Centrale de Nantes for the utilization of laser cladding machine, Gilles Carabin of the École Centrale de Nantes and Fabienne Blanchet of the University of Rennes for their technical expertise.

\section{References}

1 Y. Lin, Y. Shen, A. Liu, Y. Zhu, S. Liu, H. Jiang, Bioinspiredly fabricating the hierarchical $3 \mathrm{D}$ porous structure superhydrophobic surfaces for corrosion prevention, Mater. Des. 103 (2016) 300-307

2 D. Najjar, M. Bigerelle, A. Iost, The computer based Bootstrap method as a tool to select a relevant surface roughness parameter, Wear 254 (2003) 450-460

3 M. Bigerelle, D. Najjar, A. Iost, Multi-scale functional analyses of wear: A fractal model of the grinding process, Wear 258 (2005) 232-239

4 S. Le Cann, A. Galland, B. Rosa, T. Le Corroller, M. Pithioux, J.N. Argenson, P. Chabrand, S. Paratte, Does surface roughness influence the primary stability of acetabular cups? A numerical and experimental biomechanical evaluation, Med. Eng. Phys. 36 (2014) 1185-1190
5 B. Rosa, J.Y. Hascoet, P. Mognol, Topography modeling of laser polishing on 316 L milled surfaces, Mech. Ind. 15 (2014) $51-61$

6 A. Brient, M. Brissot, J.-C. Sangleboeuf, Service life of glass workpieces: Influence of the grinding parameters on the surface roughness and the fracture strength, Int. J. Mach. Mach. Mater. 15 (2014) 54-70

7 A. Brient, R. Laniel, M. Miroir, G. Le Goic, J.-C. Sangleboeuf, S. Samper, Multiscale topography analysis of waterjet pocketing of silica glass surfaces, Met \& Props, UNC Charlotte, USA 2-5 march 2015

8 X. Jane Jiang, D.J. Whitehouse, Technological shifts in surface metrology, CIRP Ann. Manuf. Technol. 61 (2012) $815-836$

9 S.H. Bui, B. Muralikrishnan, J. Raja, A framework for Internet-based surface texture analysis and information system, Precis. Eng. 29 (2005) 298-306

10 E. Ukar, A. Lamikiz, S. Martinez, I. Tabernero, L.N. Lopez De Lacalle, Roughness prediction on laser polished surfaces, J. Mater. Process. Technol. 212 (2012) 1305-1313

11 S.D. Philip, P. Chandramohan, P.K. Rajesh, Prediction of surface roughness in end milling operation of duplex stainless steel using response surface methodology, J. Eng. Sci. Technol. 10 (2015) 340-352

12 Y. Quinsat, S. Lavernhe, C. Lartigue, Characterization of $3 \mathrm{D}$ surface topography in 5-axis milling, Wear 271 (2011) 590-595

13 N.E. Karkalos, N.I. Galanis, A.P. Markopoulos, Surface roughness prediction for the milling of Ti-6Al-4V ELI alloy with the use of statistical and soft computing techniques, Measurement 90 (2016) 25-35

14 A. Garg, K. Tai, Comparison of regression analysis, artificial neural network and genetic programming in handling the multicollinearity problem, Proceedings of 2012 International Conference on Modelling, Identification and Control, Wuhan, China, June 24-26, 2012

15 G. Le Goic, M. Bigerelle, S. Samper, H. Favreliere, M. Pillet, Multiscale roughness analysis of engineering surfaces: A comparison of methods for the investigation of functional correlations, Mech. Syst. Signal Process. 66-67 (2016) 437-457

16 V. Belaud, S. Valette, G. Stremsdoerfer, M. Bigerelle, S. Benayoun, Wettability versus roughness: Multi-scales approach, Tribol. Int. 82 (2015) 343-349

17 R. Deltombe, K.J. Kubiac, M. Bigerelle, How to select the most relevant 3D roughness parameters of a surface, Scanning J. 36 (2014) 150-160

18 J. Marteau, M. Bigerelle, P.-E. Mazeran, S. Bouvier, Relation between roughness and processing conditions of AISI $316 \mathrm{~L}$ stainless steel treated by ultrasonic shot peening, Tribol. Int. 82 (2015) 319-329

19 J. Marteau, M. Bigerelle, Relation between surface hardening and roughness induced by ultrasonic shot peening, Tribol. Int. 83 (2015) 105-113

20 Standard ISO 25178-2, Surface texture: Areal. Parts 2: Terms, definitions and surface texture parameters, 2012

Cite this article as: B. Rosa, M. Bigerelle, A. Brient, S. Samper, Framework of models for selecting manufacturing processes and associated parameters for surface topographies, Mechanics \& Industry 20, 301 (2019) 